Check for updates

Cite this: Nanoscale Adv., 2019, 1, 1086

\title{
Development of ratiometric sensing and white light-harvesting materials based on all-copper nanoclusters $\dagger$
}

\author{
Dan Li, (D) t $^{\text {a }}$ Guannan Wang, t $^{a}$ Yongjin Peng, (D) ${ }^{a}$ Zhenhua Chen, (D) ${ }^{a}$ Xianhui Gao, ${ }^{a}$ \\ Liming Cheng ${ }^{b}$ and Xifan Mei ${ }^{* a}$
}

\begin{abstract}
Herein, we developed a special strategy for the fast sensitization of red emitting copper nanoclusters with the assistance of green emitting copper nanoclusters. Compared to most previous methods based on AIE, which do not maintain the water solubility or tiny size of nanoclusters, the charming features of the copper nanoclusters were retained after the fabrication. Furthermore, the product was employed for the detection of sulphide, which revealed its ratiometric sensing ability in water since the ratio of the intensity change for green and red emission was related to the sulphide concentration. In addition, after the addition of $\mathrm{Zn}^{2+}$, the green and red emission was either enhanced or quenched via the corresponding mechanism. This enables the facile fabrication of promising white light-harvesting materials since the species of the emitting color can be simply tuned.
\end{abstract}

Received 19th September 2018 Accepted 1st December 2018

DOI: $10.1039 / c 8 n a 00224 j$

rsc.li/nanoscale-advances

other fluorescence enhancement methods have been reported,

\section{Introduction}

Noble metal nanoclusters (NCs) have been proven as promising materials for several applications due to their molecule-like behaviors, including fluorescence properties, biocompatibility and ultra-small size. ${ }^{1}$ For the fabrication of NCs with excellent water solubility and tiny size, the use of small thiol-containing molecules to protect NCs was effective, but it was quite diffcult to obtain bright fluorescence. ${ }^{2}$ Among them, the fluorescence brightness of the red-emitting copper nanoclusters (RCuNCs) was especially weak though they tended to be favorable for optical applications. Aggregation-induced emission (AIE) resulted in fluorescence enhancement, where the vibration and rotation of NCs was inhibited and the corresponding nonradiative process was reduced. ${ }^{3}$ The fluorescence quantum yield (QY) of dispersed NCs could be boosted using various AIE strategies, such as placing NCs in a confined environment. ${ }^{4,5}$ Fluorescence enhancement was also obtained by fabricating NCs as large-size nanomaterials. ${ }^{6}$ For instance, Zhao found that the dissociation of ligands on NCs could induce the formation of aggregates and enhance the fluorescence. ${ }^{7}$ Additionally, there

\footnotetext{
${ }^{a}$ Department of Chemistry, The Key Laboratory of Medical Tissue Engineering of Liaoning Province, Jinzhou Medical University, Jinzhou, 121001, China. E-mail: danli@jzmu.edu.cn; meixifan1971@163.com

${ }^{b}$ Department of Orthopedics, Tongji Hospital Affiliated to Tongii University School of Medicine Key Laboratory of Spine \& Spinal Cord Injury Repair and Regeneration, Tongji University, Shang-hai, 200065, China

$\dagger$ Electronic supplementary information (ESI) available: Details of the characterization of the materials; factors influencing the catalysis and FRET effect. See DOI: 10.1039/c8na00224j

$\ddagger$ Both authors contributed equally to this work.
} such as encapsulating NCs in a polymer matrix or metalorganic frameworks (MOF) ${ }^{8-12}$ However, it should be noted that all these protocols actually changed the application potential of the original NCs. ${ }^{13}$ This is because were no longer well distributed in water as ultra-small size materials. ${ }^{14}$ In summary, these methods could enhance the fluorescence, but failed to reserve the special molecule-like properties of the original NCs. ${ }^{15-18}$

NCs were found to demonstrate remarkable catalytic activity for oxidization/reduction. ${ }^{19-22}$ Among them, copper nanoclusters (CuNCs) were employed to boost the rate for several types of reactions. ${ }^{23-25} \mathrm{An}$ increase in activity was realized by the introduction of different CuNCs. ${ }^{26}$ However, to the best of our knowledge, there are no reports on the use of CuNCs for the formation of other NCs. Herein, we designed a facile protocol for the synthesis of R-CuNCs via the assistance of green emitting CuNCs (G-CuNCs). In the absence of G-CuNCs, R-CuNCs were not formed until a long reaction time using dihydrolipoic acid (DHLA) as a protecting ligand. On the other hand, in the presence of G-CuNCs, R-CuNCs were fabricated using bis [tetrakis(hydroxymethyl)phosphonium] sulfate (THPS) as a reducing reagent quite fast, and their red emission was enhanced significantly via combination with appropriate amounts of $\mathrm{Cu}^{2+}$. Since this process retained the tiny size and water solubility of the NCs, it has great advantages for further applications. ${ }^{18}$ The reaction mechanism is demonstrated in Fig. 1. Initially, R-CuNCs were formed fast due to the assistance of G-CuNCs. Simultaneously, the distance between G-CuNCs and R-CuNCs was long due to the dilute solution. As R-CuNCs became concentrated via the reduction of more $\mathrm{Cu}^{2+}$, they moved closer to G-CuNCs until the chance for their electrostatic 


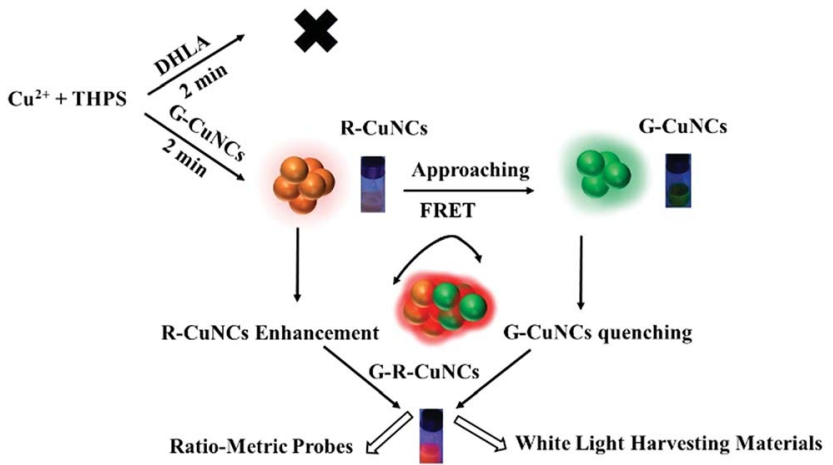

Fig. 1 Scheme for the fast formation of R-CuNCs and enhancement of their fluorescence through the assistance of G-CuNCs.

interaction was considerable. Unlike the big nanoparticles with a relatively large repulsive force, the distance between each group of NCs was extremely small since it was a "big crowd" and the electrostatic interaction as "soft aggregates" was simpler. Thereafter, a FRET system may have been constructed between the CuNCs with different emissions when their proximity was close enough.

Since the as obtained product (G-R-CuNCs) were constructed using two types of emitters, they could show different responses to analytes under certain conditions, and thus they may be applied ratiometrically either by inducing a change in the red or green emission. ${ }^{27}$ Ratiometric sensors based on NCs have been confirmed to be capable of demonstrating anti-interference effects. $^{28}$ However, most NCs are combined with additional organic fluorescence dyes or quantum dots to fabricate sensors, which involves toxic chemicals and complicated procedures. ${ }^{29,30}$ Multi-color emission was found for DNA-protected NCs and the as-prepared products facilitated the fabrication of ratiometric sensors without the introduction of other types of fluorophores. In this case, different emissions had to be excited at different wavelengths. ${ }^{31}$ Besides, DNA templates are much more expensive than common reagents, which limit their application. Herein, ratiometric sensing materials were totally constructed using cost-effective CuNCs. Subsequently, their ratiometric sensing behavior for a typical analyte $\left(\mathrm{S}^{2-}\right)$ confirmed this assumption. In addition, it was found that the red emission of the product could be enhanced, quenched or changed ratiometrically with the green emission upon the addition of $\mathrm{Zn}^{2+}$. Furthermore, was interesting to find the generation of white light during this process. These phenomena suggest that allcopper NC products can be developed as promising white light-harvesting materials because this protocol is very convenient and no dyes or other protocols were required. ${ }^{32}$

\section{Results and discussions}

\subsection{Preparation of G-CuNCs}

Initially, G-CuNCs were obtained via a facile synthetic strategy involving the modification of PVP-CuNCs with DHLA. The typical product was denoted as G-CuNCs-1, which was characterized and described in Fig. 2. As shown in Fig. 2a, its UV-Vis
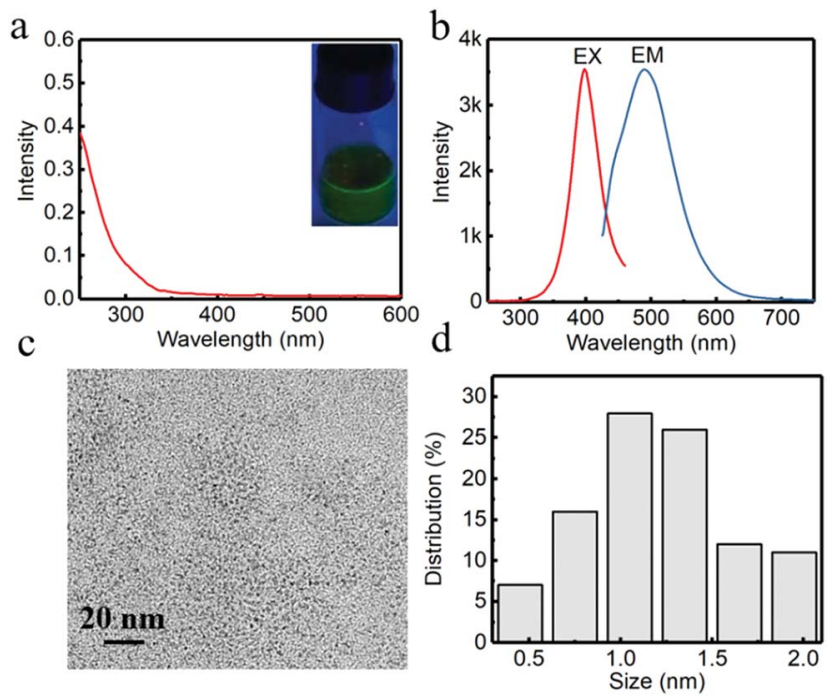

Fig. 2 (a) UV-Vis absorption spectrum of G-CuNCs-1 (inset, photograph of the sample under a $365 \mathrm{~nm}$ UV lamp), (b) fluorescence excitation (EX) and emission (EM) spectra of G-CuNCs-1 with the slit widths of $10 \mathrm{~nm}$ for both excitation and emission, (c) typical TEM image and (d) statistic size distribution of G-CuNCs-1.

absorbance spectrum demonstrated an insignificant peak that does not support the surface plasma resonance (SPR) of larger copper nanoparticles (CuNPs) (normally around $560 \mathrm{~nm}$ ). This was similar to the previously reported water-soluble CuNCs with tiny sizes. ${ }^{33}$ Meanwhile, it was demonstrated in Fig. 2a (inset) that the as-prepared G-CuNCs-1 exhibited green emission under UV light irradiation upon excitation at $365 \mathrm{~nm}$. As can be observed from Fig. 2b, G-CuNCs-1 demonstrated a fluorescence emission at $491 \mathrm{~nm}$ with the maximum excitation at $398 \mathrm{~nm}$. The QY was calculated to be about $2.46 \%$ using FITC (QY $=92 \%$ in pH 8 solution) as a reference. Furthermore, its morphology and size distribution were studied by TEM, as shown in Fig. 2c and d, respectively. It can be seen that it was approximately 0.5$2.0 \mathrm{~nm}(n=100)$ in diameter, which exhibited typical NC-level features..$^{34,35}$

\subsection{Facile formation of R-CuNCs}

It was found in previous work that R-CuNCs could be prepared using DHLA as a protecting ligand, but the reaction takes a long time. ${ }^{33}$ In the current work, we found that R-CuNCs could be obtained quite fast with the assistance of G-CuNCs. Firstly, for the evaluation of the formation tendency of R-CuNCs, different amounts of G-CuNCs were used as the assistant, as shown in Fig. 3. Initially, the fluorescence emission spectra were monitored for the synthesis of R-CuNCs without G-CuNCs. It can be seen from Fig. 3a a quite weak fluorescence with a maximum emission intensity at $c a$. $600 \mathrm{~nm}$ was only obtained a relatively high concentration of $\mathrm{Cu}^{2+}$ was combined with DHLA, THPS and $\mathrm{Cu}^{2+}$. Also, various methods were used for mixing the precursors including DHLA, THPS and $\mathrm{Cu}^{2+}$; however, still no RCuNCs with satisfactory red emitting response could be observed within a short time. This revealed that it was difficult to obtain water-soluble R-CuNCs with excellent fluorescence 

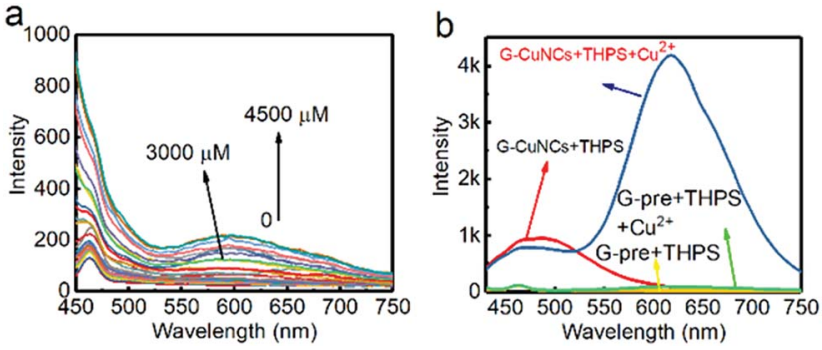

Fig. 3 (a) Fluorescence emission spectra of the mixtures with the combination of the protecting ligands (DHLA), reducing reagent (THPS), and various amounts of $\mathrm{Cu}^{2+}$. (b) Fluorescence response for the fabrication of R-CuNCs in the absence (green and yellow lines) and presence (red and blue lines) of G-CuNCs (100 $\mu \mathrm{L})$ using $40 \mu \mathrm{L}$ of $0.079 \%$ THPS after the addition of 0 (red and yellow lines) and $100 \mu \mathrm{M}$ (green and blue line) of $\mathrm{Cu}^{2+}$. G-pre + THPS indicates the medium underwent the same treatments for the preparation of G-CuNCs in the absence of $\mathrm{Cu}^{2+}$.

behaviors quite fast using the one-pot synthetic strategy without additional assistance. However, it can be seen from Fig. $3 \mathrm{~b}$ that remarkable red emission was obtained within two minutes using the same synthetic protocol in the presence of G-CuNCs. In addition, specific fluorescence emission spectra for R-CuNCs were even exhibited with the combination of a relatively low concentration of $\mathrm{Cu}^{2+}$. On the other hand, no red emission was observed using such low amounts of $\mathrm{Cu}^{2+}$ in the absence of GCuNCs. Apparently, R-CuNCs were formed much more easily due to the additional assistance. Thus, to deeply understand what assisted the formation of R-CuNCs (the entire G-CuNCs or the pre-synthesis materials for G-CuNCs), it was worth investigating whether R-CuNCs could form quite fast with the assistance of other compounds. Subsequently, $\mathrm{Cu}^{2+}$ was combined with a mixture treated with the same protocol as that for the preparation of G-CuNCs without the involvement of the copper precursor (see Fig. 3b, green and yellow lines). It should be noted that PVP, L-ascorbic acid and DHLA were present in the mixture (G-pre). However, no fluorescence response corresponding to the generation of R-CuNCs was observed within a short time using a same strategy. This revealed that the fast formation of R-CuNCs could not occur until G-CuNCs were used.

This phenomenon was further evaluated with different amounts of G-CuNCs, as shown in Fig. 4. For the investigations, $500 \mu \mathrm{L}$ of $0.079 \%$ THPS was used as the reducing agent. The mixture was prepared in the presence of different amounts of G-CuNCs with a fixed volume. After the combination with different amounts of $\mathrm{Cu}^{2+}$, the fluorescence emission spectra were recorded. Meanwhile, a product (R-CuNCs-0) in the absence of G-CuNCs was prepared with a similarly long reaction time. It was found that the most intense fluorescence intensity was not generated until overnight reaction. After the synthesis, the product was characterized and described in Fig. S1. $\dagger$ It can be seen from Fig. S1 $\uparrow$ that the fluorescence was quite weak. However, when 25-50 $\mu \mathrm{L}$ of G-CuNCs was employed, the fluorescence was obtained within two minutes, as shown in Fig. 4a and b. Since the fluorescence emission
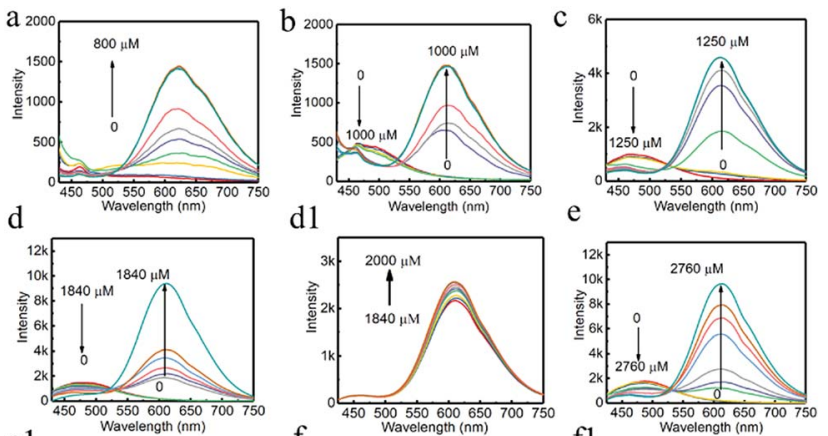

e1
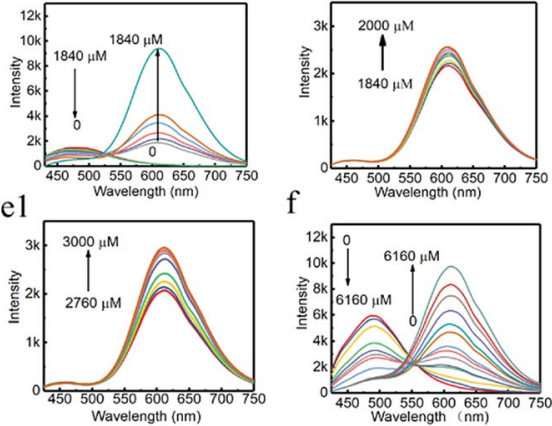

$\mathrm{f}$
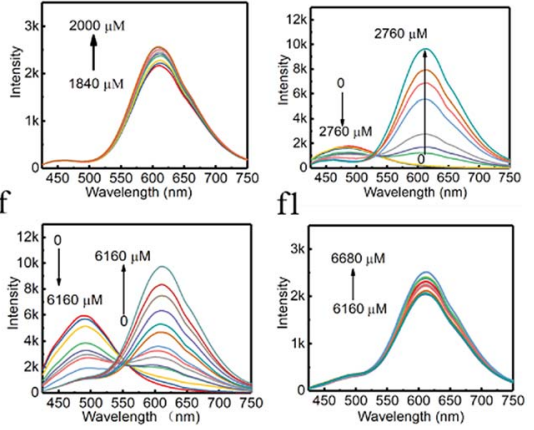

Fig. 4 Fluorescence emission spectra of the generation of G-RCuNCs as a function of the amount of G-CuNCs, (a) $25 \mu \mathrm{L}$, (b) $50 \mu \mathrm{L}$, (c) $100 \mu \mathrm{L}$, (d) $150 \mu \mathrm{L}$, (e) $250 \mu \mathrm{L}$, and (f) $1000 \mu \mathrm{L}$ with the slit widths of $10 \mathrm{~nm}$ for both excitation and emission. (d1) $(150 \mu \mathrm{L})$, (e1) $(250 \mu \mathrm{L})$, and (f1) $(1000 \mu \mathrm{L}$ ) were with the slit widths of $10 \mathrm{~nm}$ for excitation and $5 \mathrm{~nm}$ for emission.

spectra were demonstrated using the same measurement conditions as that in Fig. $\mathrm{S} 1, \uparrow$ it could be concluded that the asformed G-R-CuNCs exhibited the brightest fluorescence in Fig. $4 \mathrm{a}$ and $\mathrm{b}$. At this stage, the enhanced fluorescence was possibly caused by the fast reaction, which facilitated the formation of brighter NCs due the acceleration of the nucleation of NCs and vice versa. ${ }^{36}$ Since ab increase in the content of G-CuNCs did not enhance the fluorescence emission intensity of R-CuNCs dramatically, it seemed the presence of G-CuNCs was only capable of effectively boosting the formation rate of R-CuNCs. ${ }^{33}$ However, it was further found that the fluorescence emission intensity at $610 \mathrm{~nm}$ could be significantly enhanced while that at $490 \mathrm{~nm}$ decreased if $100 \mu \mathrm{L}-1000 \mu \mathrm{L}$ of G-CuNCs was applied, as shown in Fig. 4c-f. This revealed that the presence of G-CuNCs at higher concentrations played a more important role than just assistants for accelerating the formation rate of R-CuNCs. The enhancement and quenching of the fluorescence at $610 \mathrm{~nm}$ and $490 \mathrm{~nm}$, respectively, agreed with the typical FRET phenomenon, where both donor and acceptor existed. ${ }^{37}$ However, for the occurrence of FRET, the distance between the donor and acceptor is important. By using low amounts of G-CuNCs, the NCs were relatively isolated from each other in the solution. This meant that the chance for the approximation or interaction from G-CuNCs to R-CuNCs was low. At the same time, it can be seen from Fig. 4a and $\mathrm{b}$ that the emission intensity at $490 \mathrm{~nm}$ for G-CuNCs was quite weak, which could hardly play a role as a donor. On the other hand, when enough G-CuNCs were present in the system, the intensity of the green emission was stronger. Meanwhile, the occupation of more space due to the high concentrations facilitated G-CuNCs approaching R-CuNCs. Then, the opportunities for proximity between the promising donor and acceptor would be greatly increased. Therefore, the problem for realizing the FRET process could possibly be overcome. 
According to the results from Fig. 4d-f, all the emission spectra for G-R-CuNCs were significantly enhanced after the combination with enough $\mathrm{Cu}^{2+}$. The typical fluorescence change as a function of $\mathrm{Cu}^{2+}$ in detail was also described in Fig. S2. $\dagger$ It can be seen that the emission intensity at $610 \mathrm{~nm}$ did not increase linearly as a function of $\mathrm{Cu}^{2+}$ from low concentration to high concentration. On the contrary, the fluorescence exhibited little change initially and dramatically jumped at a certain concentration. This was not surprising since the RCuNCs formed at first possibly did not influence the fluorescence behavior of G-CuNCs. Also, the fluorescence emission demonstrated overlapping behavior. A scheme is presented in Fig. S3† to demonstrate the increasing approximation of GCuNCs and R-CuNCs during the latter process. Similar to the requirement of relatively large amounts of G-CuNCs, the opportunity for approximation was not realized until relatively large amounts of R-CuNCs were formed. It should be noted from Fig. 4d1-f1 that the highest emission intensity for RCuNCs was almost same when the amounts of G-CuNCs were different. This was quite different from the AIE phenomenon that the enhancement of fluorescence increases as a function of the induced materials when their content is not high. ${ }^{38}$

Meanwhile, it was found that much larger amounts of $\mathrm{Cu}^{2+}$ were required for the generation of the lowest response of red emission when larger amounts of G-CuNCs were applied. This was caused by the complexing effect of DHLA. If the ratio of $\mathrm{Cu}^{2+}$ was not considerable, it would complex with DHLA rather than transfer to $\mathrm{Cu}^{0}$ by reduction with THPS. Thereafter, no RCuNCs could be formed to generate red emission until DHLA was complexed with enough $\mathrm{Cu}^{2+}$. Besides this, the increasing requirement of higher amounts of $\mathrm{Cu}^{2+}$ to reach both the strongest brightness and lowest response for red emission was found as a function of THPS within a certain range, as shown in Fig. S4 and Table S1. $\dagger$ It was observed that only after enough $\mathrm{Cu}^{2+}$ was combined, additional $\mathrm{Cu}^{2+}$ would sensitize the fluorescence of R-CuNCs. This indicated both THPS and DHLA play the role of complexing reagents because previous work also found that the reducing reagent functioned as a complexing reagent as well. ${ }^{39}$ Additionally, in the absence of THPS, the fluorescence enhancement at $610 \mathrm{~nm}$ was observed as a function of $\mathrm{Cu}^{2+}$ (Fig. S4a $\dagger$ ). This indicated that the adsorbed LAA on the surface of G-CuNCs had the ability to reduce $\mathrm{Cu}^{2+}$ to $\mathrm{Cu}^{0}$, but the as obtained product exhibited very weak fluorescence behaviors. Thus, the application of THPS as an additional reducing reagent is necessary to obtain G-R-CuNCs with excellent features.

G-CuNCs could significantly enhance the formation rate for the generation of R-CuNCs, which appears to be similar to a catalytic process according to the above results. However, G$\mathrm{CuNCs}$ had to be reserved after they assisted the formation of R-CuNCs. Based on the phenomena in Fig. 4f, the feature of green emission for G-CuNCs was not observed anymore after the addition of high amounts of $\mathrm{Cu}^{2+}$, which indicated G-CuNCs were probably used up since their green emission was almost quenched. However, the formation of R-CuNCs enabled the quenching of the green fluorescence if FRET occurred. In that case, we could not observe the fluorescence of G-CuNCs even though they were still present in the colloid. On the other hand, if the FRET was truly the reason that we could not observe the fluorescence of G-CuNCs after the generation of R-CuNCs, the green emission should be recovered to a certain extent when red emission was destroyed or masked.

To investigate the phenomena and eliminate the possible interferences for the enhancement/quenching behaviors, GCuNCs without the protection of PVP were prepared (GCuNCs-2). Subsequently, they were used as assistants for the fabrication of R-CuNCs (R-CuNCs-2). The typical process for the generation and inhibition of red emission is described in Fig. S5. $\uparrow$ Initially, it could be observed the fluorescence of GCuNCs-2 was quenched as a function of $\mathrm{Cu}^{2+}$ due to the formation of R-CuNCs-2 (Fig. S5a $\dagger$ ). This was similar to the fluorescence evolution behavior in Fig. 4f. Subsequently, we tried to select a reagent that would mask the fluorescence of RCuNCs, but would not be strong enough to inhibit the fluorescence of G-CuNCs. Since thio-containing molecules would easily react with both G-CuNCs and R-CuNCs at high concentrations, the amino acid L-histidine was explored. It was reported R-CuNCs could be quenched after the combination of a relatively high concentration of L-histidine through the formation of a more stable $\mathrm{His} / \mathrm{Cu}^{2+}$ complex due to the participation of the imidazole ring of His. ${ }^{\mathbf{4 0}}$ Herein, the masking of R-CuNCs was demonstrated since the red emission was quenched, as shown in Fig. S5b. $\dagger$ Meanwhile, it was interesting to observe that the green emission of G-CuNCs-2 was recovered. However, the emission peak in Fig. $\mathrm{S} 5 \mathrm{~b} \dagger$ became broader and its relative intensity was not exactly the same as that in Fig. S5a. $\dagger$ This indicated the possible occurrence of a recovery process, but it was not completely reversible. Notwithstanding, since the green emission could not be generated from the other applied materials, but only CuNCs, it can be concluded that the presence of G-CuNCs was significant. This indicated G-CuNCs may still exist after the formation of R-CuNCs although their feature were not observed. Therefore, the enhanced formation rate for R-CuNCs was possibly caused by the involvement of GCuNCs as a catalyst. However, since the generation and enhancement of the red emission were apparently controlled by more complicated processes, G-CuNCs were considered as an 'assistant' rather than a catalyst. Although G-CuNCs-2 could also be used for assisting the formation of R-CuNCs, it was not as stable as the PVP-protected G-CuNCs. Besides, it was found that large amounts of G-CuNCs-2 were required for realizing a similar process, which is possibly attributed to the different surface states, sizes, and other conditions. ${ }^{41}$ As a result, they were only employed for understanding the reaction mechanism. For further applications, the PVP-protected G-CuNCs were employed.

For fabrication of the red-emitting product, a typical sample (G-R-CuNCs-1) was obtained using $200 \mu \mathrm{L}$ of G-CuNCs as the assistant and $500 \mu \mathrm{L}$ of $0.079 \%$ THPS as a reducing reagent. After the combination with enough $\mathrm{Cu}^{2+}$, the product with the highest fluorescence enhancement was characterized and described in Fig. 5. As shown in Fig. 5a, the UV-Vis absorption spectrum of G-R-CuNCs-1 exhibited an insignificant peak, which indicated that the molecule-like behavior of CuNCs was 


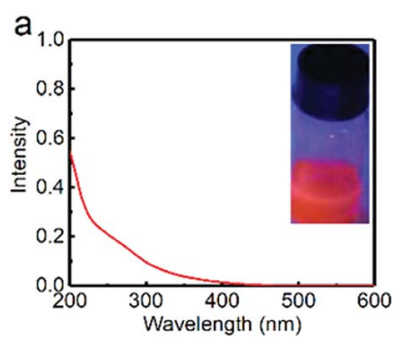

c
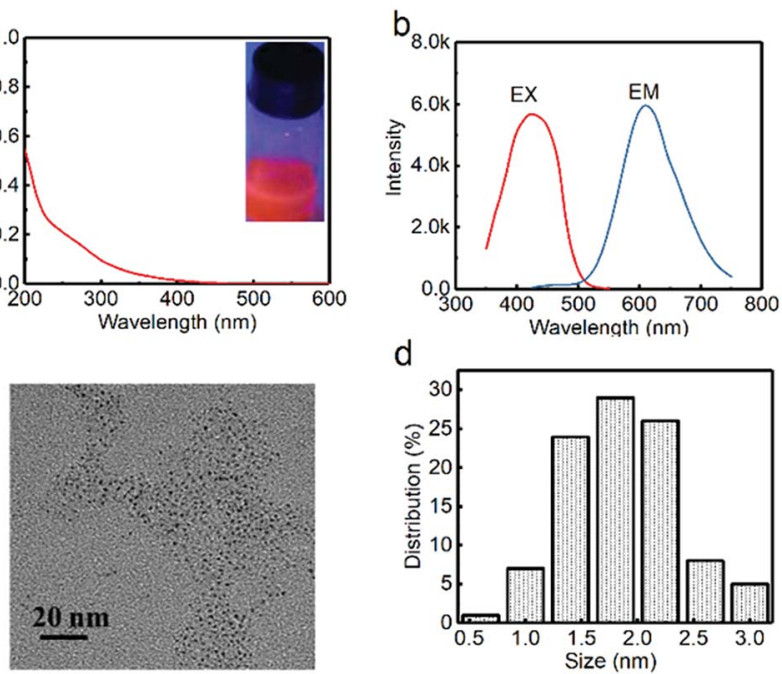

d

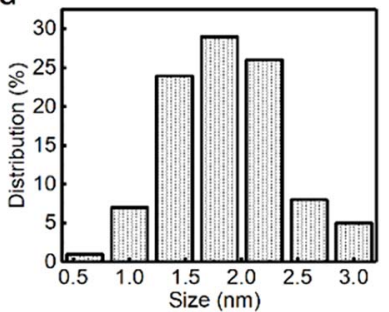

Fig. 5 (a) UV-Vis absorption spectrum of G-R-CuNCs-1, (b) fluorescence excitation (EX) and emission (EM) spectra of G-R-CuNCs-1 with the slit widths of $10 \mathrm{~nm}$ for excitation and $5 \mathrm{~nm}$ for emission, (c) typical TEM images (d) and statistic size distributions of G-R-CuNCs-1.

not destroyed. ${ }^{19}$ A strong red emission was observed with its maximum peak at $610 \mathrm{~nm}$ in the fluorescence spectrum when the sample was irradiated with $424 \mathrm{~nm}$. It also exhibited a bright red emission under UV light at $365 \mathrm{~nm}$ (inset of Fig. 5a). The QY was calculated to be $4.83 \%$ using acridine yellow $(47 \%$ in ethanol) as a reference. As shown in Fig. $5 \mathrm{c}$ and d, the G-RCuNCs- 1 were approximately $0.5-3.0 \mathrm{~nm}(n=100)$, and were dispersed as uniformly as the G-CuNCs in Fig. 2c. Table S2 $\dagger$ presents a comparison of the synthetic conditions for the fabrication of the water-soluble R-CuNCs. It can be seen that our work show great advantages for the development of watersoluble products for CuNCs with fast reaction time.

\subsection{Comparison of the properties of the CuNCs}

For further understanding the relationship between the modified product of G-R-CuNCs-1, original G-CuNCs-1 and the slowly formed R-CuNCs-0, other characterizations including DLS, FTIR and XPS were performed, as shown in Fig. S7-S9, $\uparrow$ respectively. It can be seen from Fig. $\mathrm{S} 7 \dagger$ that the DLS study for the CuNCs demonstrated small sizes, which were similar to the TEM study. The zeta potentials of G-CuNCs-1, G-R-CuNCs-1 and R-CuNCs-0 were determined to be $c a$. $-29.6 \mathrm{mV},-38.1 \mathrm{mV}$, and $-25.2 \mathrm{mV}$ respectively, revealing that the surface of all these CuNCs were negatively charged. This indicated the CuNCs could maintain excellent stability due to the electrostatic repulsion effects. The surface groups of the synthesized CuNCs were further investigated using FT-IR, as shown in Fig. S8. $\dagger$ The typical S-H stretching of DHLA in the range of ca. 2500$2600 \mathrm{~cm}^{-1}$ was not observed for all the products of CuNCs. This indicated that the surface of the CuNCs were capped by the S-H group from the ligand. ${ }^{42}$ Furthermore, XPS analysis was employed to investigate the oxidation state of $\mathrm{Cu}$, as shown in Fig. S9. $\uparrow$ The peaks at $932.4 \mathrm{eV}$ and $952.1 \mathrm{eV}$ can be ascribed to the binding energy of the $2 \mathrm{p}_{3 / 2}$ and $2 \mathrm{p}_{1 / 2}$ electrons of the $\mathrm{Cu}$ atoms (Fig. S9a $\dagger$ ), respectively. ${ }^{12,19}$ Meanwhile, the XPS spectrum shows a more positive peak for G-R-CuNCs-1 at $952.3 \mathrm{eV}$, which can be assigned to the binding energy of the $\mathrm{Cu} 2 \mathrm{p}_{1 / 2}$ electrons of metallic copper for both $\mathrm{Cu}(0)$ and $\mathrm{Cu}(\mathrm{I})$ (Fig. S9c $\dagger$ ). ${ }^{\mathbf{2 4 , 4 3 , 4 4}}$ These results suggest that more electrons were lost for $\mathrm{Cu}$ in G-R-CuNCs-1 compared to G-CuNCs-1. Furthermore, it could be observed that $\mathrm{Cu} 2 \mathrm{p}$ for R-CuNCs-0 showed even more positive peaks for both $\mathrm{Cu} 2 \mathrm{p}_{3 / 2}$ and $\mathrm{Cu} 2 \mathrm{p}_{1 / 2}$ at $932.7 \mathrm{eV}$ and $952.6 \mathrm{eV}$, respectively (Fig. S9e $\dagger$ ). These results revealed that R-CuNCs- 0 tended to exhibit more positive values than G-CuNCs- $1 .{ }^{45}$ On the other hand, the XPS survey spectra in Fig. S9b, $d$ and $f \dagger$ for the all the products described the presence of C, S and O, which belonged to DHLA. This agreed with the FTIR investigation that CuNCs were well protected by the DHLA ligand.

\subsection{Mechanism analysis}

To understand the possible mechanisms for the fluorescence change in G-CuNCs due to the generation of R-CuNCs, we proposed that FRET may control the process. The efficiency for FRET $\left(E_{\text {FRET }}\right)$ was initially calculated according to Förster's theory:

$$
E_{\mathrm{FRET}}=\frac{R_{0}{ }^{6}}{{R_{0}}^{6}+r_{0}{ }^{6}}=1-\frac{F_{\mathrm{DA}}}{F_{\mathrm{D}}}
$$

where, $R_{0}$ is the critical distance at which the energy transfer efficiency is $50 \%$ and $r_{0}$ is the distance between the donor and the acceptor. Meanwhile, $F_{\mathrm{DA}}$ and $F_{\mathrm{D}}$ represent the fluorescence intensity of the donor in the presence and absence of the acceptor, respectively. For instance, the fluorescence intensity of a typical process at the peak $(490 \mathrm{~nm})$ after the formation of G-R-CuNCs for $F_{\mathrm{D}}$ and $F_{\mathrm{DA}}$ was 937.8 and 2262.4 (as shown in Fig. S2 $\dagger$ ), respectively. Then, based on the above formula, $E_{\text {FRET }}$ could be calculated as $58.5 \%$. In theory, the distance between the two CuNCs could be as short as the size of the two DHLA molecules between them ca. $2.6 \mathrm{~nm}$, see Fig. S10. $\dagger$ Then $R_{0}$ could be calculated as $2.7 \mathrm{~nm}$ according to the above formula. In this close proximity, G-CuNCs could emit a virtual photon, which would then be absorbed by R-CuNCs. Then, after excitation, we observed a significant fluorescence enhancement for the red emission and quenching for the green emission.

To obtain more information on this process, the fluorescence decay curves for the typical transformation of G-CuNCs to G-R-CuNCs were measured, as shown in Fig. S11. $\uparrow$ Herein, the lifetimes of the donor and the as-obtained products were investigated. The curves could all be fitted by a threeexponential function, with three components. The triple exponential decay curve for the lifetime study can be described as follows:

$$
I(t)=A_{1} \exp \left(\frac{-t}{\tau_{1}}\right)+A_{2} \exp \left(\frac{-t}{\tau_{2}}\right)+A_{3} \exp \left(\frac{-t}{\tau_{3}}\right)
$$

$E_{\text {FRET }}$ is obtained from the donor exciton lifetime upon interaction with the acceptor, where $\tau_{\mathrm{DA}}$ (G-CuNCs-b) is the fluorescence lifetime of the donor in the presence of the 
acceptor and $\tau_{\mathrm{D}}$ (G-CuNCs-a) is the fluorescence lifetime of the donor alone.

$$
E_{\mathrm{FRET}}=1-\frac{\tau_{\mathrm{DA}}}{\tau_{\mathrm{D}}}
$$

The lifetime results were described in Table $\mathrm{S} 3 \dagger$ and the $E_{\text {FRET }}$ was calculated to be $47.2 \%$. Herein, the as obtained $E_{\text {FRET }}$ was not optimized. It could be tuned via the appropriate combination of G-CuNCs, reducing reagent (THPS), and $\mathrm{Cu}^{2+}$. Thus, it is necessary for the details of this process to be investigated in the future.

Unlike AuNCs, the absorption peak for the water-soluble CuNCs was not quite obvious without theoretical calculation. $^{46,47}$ Normally, when some materials were excited to a higher state, they tended to end up in the lowest excited state and then emitted radiation. In this case, the excitation peak agreed well with the absorption peak. For determination of the typical band gap, the absorption was analyzed using the excitation peak as a reference. Then, the absorption edge was estimated as $390 \mathrm{~nm}$ (excitation, $398 \mathrm{~nm}$ ) and $412 \mathrm{~nm}$ (excitation, $424 \mathrm{~nm}$ ), respectively according to Fig. S12. $\dagger$ Furthermore, based on the following equation, it was concluded that GCuNCs and R-CuNCs exhibited a band gap of $c a .3 .2 \mathrm{eV}$ (Fig.S12a $\dagger$ ) and $c a .3 .0 \mathrm{eV}$ (Fig. S12b $\dagger$ ), respectively.

$$
E_{\mathrm{g}}=\frac{1240}{\lambda}
$$

Meanwhile, the overlap of the emission spectra of G-CuNCs and the excitation spectra of R-CuNCs was analyzed, as shown in Fig. S12c. $\dagger$ Using the Origin software, the overlapping potion was calculated to be more than $42 \%$ for both CuNCs. For quantum dots with similar structures, it was found that energy transfer could occur if the acceptors had a bandgap smaller than that of the donors of $55 \mathrm{meV} .^{48}$ Additionally, for quantum dots emitting in the NIR, the energy difference should be bigger than $c a .59 \mathrm{meV} .^{49}$ The CuNCs exhibited quite similar fluorescence properties to quantum dots. Thus, it was reasonable to assume that more than $200 \mathrm{meV}$ should be enough for the energy transfer between the two NCs.

Additionally, a cyclic voltammetry study was conducted to estimate the valence and conduction band energy levels of the CuNCs (see Fig. S13†). The CuNCs were not as stable as AuNCs in water. With an increase or decrease in potential, the dissociation of the protecting ligands may interfere with the observation. Thus, to avoid unexpected errors, we calculated the valence and conduction band energy levels from the onset oxidation $\left(E_{\mathrm{ox}}\right)$ and reduction $\left(E_{\mathrm{red}}\right)$ potentials using a previously reported paper as a reference. As the structures of the protecting ligands for L-cysteine and DHLA were similar, we assumed our CuNCs would exhibit similar electrochemical behaviour to their CuNCs. ${ }^{50}$ Thereafter, according to Fig. S13a, $\dagger$ the onset $E_{\text {ox }}$ and $E_{\text {red }}$ were calculated to be $0.364 \mathrm{~V}$ and $-1.0 \mathrm{~V}$ and the estimated $\mathrm{VB}$ and $\mathrm{CB}$ energy levels were $-5.16 \mathrm{eV}$ and $-3.8 \mathrm{eV}$ for GCuNCs, respectively. Meanwhile, based on Fig. S13b, $\uparrow$ the estimated VB and CB energy levels were calculated to be $-5.12 \mathrm{~V}$ and $-3.82 \mathrm{~V}$, respectively. This agreed well with the $\mathrm{VB}$ and $\mathrm{CB}$ energy levels of the L-cysteine-protected CuNCs, which were $-5.11 \mathrm{eV}$ and $-3.7 \mathrm{eV}$, respectively. The gap between G-CuNCs and R-CuNCs suggested that the electrons could transfer from a higher to the lower energy level.

To obtain more information on these CuNCs, ESI-MS was employed for the characterization of the unknown molecular species. Negative-ion mode ESI was used since it has been employed to appropriately characterize other CuNCs. ${ }^{51} \mathrm{~A}$ representative ESI-MS investigation of the CuNCs is shown in Fig. S14. $\dagger$ It can be seen from Fig. S14a $\uparrow$ that the dominant ions in the mass spectra correspond to different combinations of copper and DHLA. There were several types of CuNCs with different atomic numbers. For example, $\mathrm{Cu}_{2}, \mathrm{Cu}_{3}, \mathrm{Cu}_{4}$, and $\mathrm{Cu}$ (DHLA $)_{x}$ complexes were observed for G-CuNCs. On the other hand, other species of NCs containing $\mathrm{Cu}_{6}$ and $\mathrm{Cu}_{7}$ were present for G-R-CuNCs-1. It should be noted that the Cu(DHLA) $)_{x}$ complex disappeared in G-CuNCs-1 (Fig. S14b†). This indicated that the green emission was possibly quenched partly due to the transformation of the $\mathrm{Cu}$ (DHLA) $)_{x}$ complex to $\mathrm{Cu}_{6}$ and $\mathrm{Cu}_{7}$. Meanwhile, the energy transfer from $\mathrm{Cu}_{2}, \mathrm{Cu}_{3}$ and $\mathrm{Cu}_{4}$ to $\mathrm{Cu}_{6}$ and $\mathrm{Cu}_{7}$ should play an important role for this fluorescence enhancement process. Interestingly, it was observed that $\mathrm{Cu}_{6}$ was not present in the slowly formed R-CuNCs-0 (Fig. S14c†). This revealed that more complicated processes could be involved in the generation of G-R-CuNCs-1. For instance, $\mathrm{Cu}_{6}$ may transform into $\mathrm{Cu}_{4}$ and $\mathrm{Cu}_{2}$, while $\mathrm{Cu}_{7}$ could transform into $\mathrm{Cu}_{3}$ and $\mathrm{Cu}_{4}$ in the ESI mode. Based on the ESI-MS, the CuNCs should be smaller than $1 \mathrm{~nm}$ according to the structure analysis in Fig. S15. $\dagger$ However, larger particles were observed in both Fig. $2 \mathrm{c}$ and $5 \mathrm{c}$. This was caused by the aggregation associated with the organic ligand-capped small particles in the presence of the $200 \mathrm{keV}$ strong electron beam. ${ }^{52}$

Besides this, we investigated many theoretical modes to predict the accurate structures of the CuNCs. However, we are not sure that the simulated structure is the optimized one since the system is a mixture, which is quite complicated. The credible way to simulate the structure of a sample would be based on the formation of a single crystal. However, the fabrication of crystals for CuNCs capped by flexible ligands is extremely difficult. ${ }^{53}$ Thus, further effort is required to obtain more pure materials in combination with the calculation.

\subsection{Ratiometric sensing applications}

Combined systems with two types of fluorophores are normally excellent candidates for sensing because each emission can be employed to exhibit a response to analytes. For instance, $\mathrm{S}^{2-}$ could sensitively quench R-CuNCs and a turn-off sensor was developed. ${ }^{14}$ On the other hand, we found a much higher concentration of $\mathrm{S}^{2-}$ was required to quench G-CuNCs. This indicated a facile ratiometric probe could be fabricated based on the current system since its response would be different from a turn-off sensor. It was demonstrated that the product with the most significant enhancement was not obtained until large amounts of THPS and $\mathrm{Cu}^{2+}$ were employed. Normally, employing a lower concentration of fluorescence sensor will result in 
high sensitivity because fewer analytes are required to react or function with it. Meanwhile, the change in the fluorescence intensity will be more significant in the presence of same amount of analyte. It was proven in Fig. $\mathrm{S} 4 \dagger$ that the requirement of $\mathrm{Cu}^{2+}$ increased as a function of THPS by employing the same amount of G-CuNCs. Thus, the tendency for the required copper in the product will be the same as that of THPS. Then, the detection of $\mathrm{S}^{2-}$ using the sensor obtained with different amounts of THPS was investigated and demonstrated in Fig. S16. $\dagger$ It can be clearly observed that the fluorescence intensity at $610 \mathrm{~nm}$ changed less significantly by using greater amounts of THPS. This revealed that high sensitivity could be obtained by using low amounts of $\mathrm{Cu}^{2+}$. However, the weak brightness of a sensor suffers from high signal-noise interferences. Thus, for balancing both the sensitivity and interferences, $150 \mu \mathrm{L}$ of G-CuNCs and $20 \mu \mathrm{L}$ of $0.079 \%$ THPS were chosen to fabricate the sensor. As a result, a sensing product (GR-CuNCs-3) was obtained after combination with $\mathrm{Cu}^{2+}$. Herein, the amount of applied THPS was relatively low, but the fluorescence of the product was strong enough for analysis, which exhibited both excellent sensitivity and insignificant signalnoise interferences.

To evaluate the sensitivity and selectivity of the sensor for the determination of $\mathrm{S}^{2-}$, the linear relationship and frequently encountered interferences were studied, as shown in Fig. 6. Fig. 6a depicts the titration of G-R-CuNCs-3 with respect to different concentrations of $\mathrm{S}^{2-}$. It can be observed that the fluorescence intensity at $610 \mathrm{~nm}$ decreased with an increase in $\mathrm{S}^{2-}$, revealing that $\mathrm{S}^{2-}$ effectively quenched the red emitting species (R-CuNCs-3). To understand the change in NCs in the presence of $\mathrm{S}^{2-}$, the TEM image was obtained, as shown in Fig. S17. $\dagger$ It can be seen that large aggregates were present. This

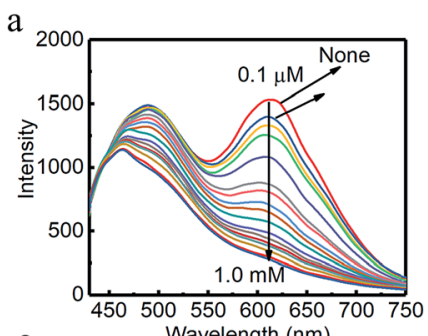

C
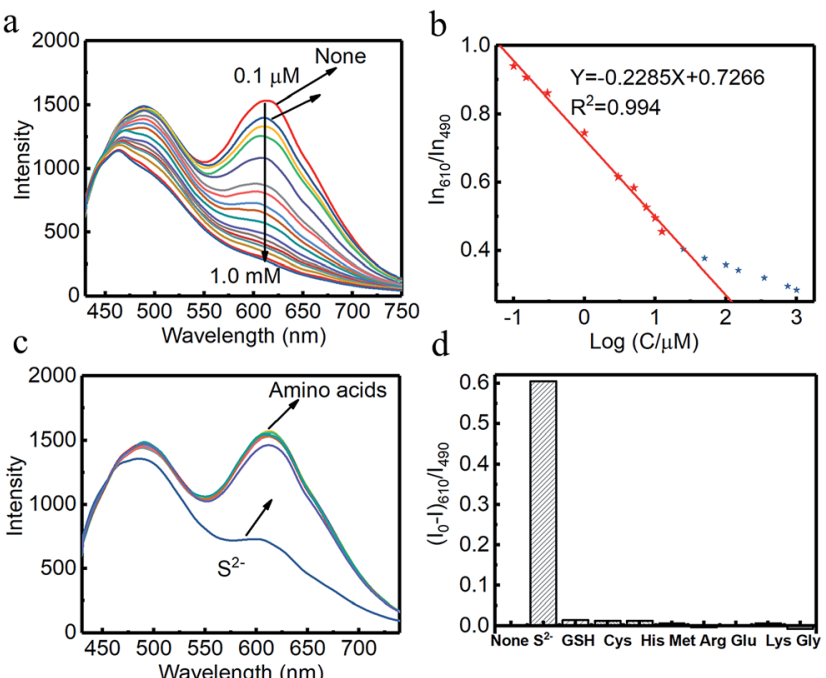

Fig. 6 (a) Fluorescence emission spectra of the sensing system in the presence of different amounts of $\mathrm{S}^{2-}$, (b) ratio of the fluorescence emission intensity at $610 \mathrm{~nm}$ to $490 \mathrm{~nm}$ (excitation, $400 \mathrm{~nm}$ ) as a function of $\log \mathrm{S}^{2-}$ concentration, (c) fluorescence emission spectra of the sensing system in the presence of $10 \mu \mathrm{M}$ of amino acid and $\mathrm{S}^{2-}$ and (d) selectivity of the sensor for $\mathrm{S}^{2-}$ over common amino acids (GSH was also used). is attributed to the exchange reaction between the surface ligands (DHLA) of R-CuNCs-3 and $\mathrm{S}^{2-}$, which enabled the formation of large $\mathrm{Cu}_{2} \mathrm{~S}$ precipitates. On the other hand, it can be observed that the intensity at $490 \mathrm{~nm}$ changed much more gently as a function of $\mathrm{S}^{2-}$ than that at $610 \mathrm{~nm}$ because GCuNCs were normally more stable. The ratio of the different responses between the intensity at $610 \mathrm{~nm}$ and $490 \mathrm{~nm}\left(I_{610}\right)$ $\left.I_{490}\right)$ revealed a good linear relationship with $\log (C / \mu \mathrm{M})$ of $\mathrm{S}^{2-}$. This enabled the signal of the sensor to change ratiometrically as a function of $\mathrm{S}^{2-}$ at both low and high concentrations. To further explore the feasibility to use the G-R-CuNCs-3 as an $\mathrm{S}^{2-}$ probe, its calibration plot was investigated. The $Y$ axis was established using the intensity ratio for the emission intensity at $610 \mathrm{~nm}\left(I_{610}\right)$ and $490 \mathrm{~nm}\left(I_{490}\right)$. Meanwhile, the logarithm of the concentration $(\log (C / \mu \mathrm{M}))$ was employed as the $X$ axis. The regression equation was $Y=-2.285 X+0.7266$, with the correlation coefficient of 0.994 (Fig. 6b). Additionally, the limits of detection were measured to be much smaller than $100 \mathrm{nM}$ based on the smallest fluorescence response upon the combination of $\mathrm{S}^{2-}$. For the interference analysis, $10 \mu \mathrm{M}$ of common amino acids and glutathione (GSH), which usually exist in biological media, were investigated. From the results presented in Fig. $6 \mathrm{c}$ and d, it can be concluded that the fluorescence response for other interferences was not comparable to that of $\mathrm{S}^{2-}$ at the same level in the current system. However, the mercapto amino acid GSH influenced the red emission at higher concentrations for CuNCs and the other amino acids quenched R-CuNCs at even higher concentrations. Nevertheless, their effects cannot compare with the quenching ability of $\mathrm{S}^{2-}$ at trace levels. Besides, other common cations and biomolecules with comparable concentrations did not show significant quenching behaviors for the sensing of $\mathrm{S}^{2-}$ using the current medium, as shown in Fig. S18. $\dagger$

The proposed sensor was applied to detect $S^{2-}$ in chicken blood serum samples and the analytical results are presented in Table S4. $\dagger$ It was found that the recovery ratio of $\mathrm{S}^{2-}$ reached 95.3-113\% with a relative standard deviation (RSD) of 0.94$1.18 \%$, which demonstrated the excellent analytical performance of the ratiometric sensor for the detection of $\mathrm{S}^{2-}$ in aqueous samples. The results were also compared to that of previously reported ratiometric sensors based on NCs, as shown in Table S5. $\dagger$ It can be observed that the as-fabricated sensor required more convenient protocols and lower cost, but its sensing performance was as excellent as the other sensors. The comparison indicated the as-obtained CuNC system is a promising sensing material.

\subsection{White light harvesting system}

Since different turn-off behaviors were exhibited for $\mathrm{S}^{2-}$ in the system for G-CuNCs and R-CuNCs, it was worth investigating whether the green emission or red emission could be induced differently by some other factors. Firstly, the requirement of low amounts of $\mathrm{Cu}^{2+}$ was designed for the fabrication of the materials based on our strategy. Then, some products were synthesized using $500 \mu \mathrm{L}$ of G-CuNCs as the catalyst and $50 \mu \mathrm{L}$ of $0.079 \%$ THPS as the reducing reagent. After adding $100 \mu \mathrm{L}$ of 
$0.01 \mathrm{M} \mathrm{Cu}^{2+}$, the product (G-R-CuNCs-4) was formed. Furthermore, the fluorescence emission spectra of G-R-CuNCs-4 was demonstrated as a function of $\mathrm{Zn}^{2+}$ in Fig. 7. It was interesting to find that the emission peak located at $490 \mathrm{~nm}$ significantly increase with a slight blue shift upon the addition of $\mathrm{Zn}^{2+}$, while the emission peak at $610 \mathrm{~nm}$ slightly decreased, as shown in Fig. 7a. The CIE1931 chromaticity coordinate calculation was performed to illustrate the details of the light tuning in the system. As displayed in Fig. $7 \mathrm{~b}$ and S19† (the corresponding picture), it can be seen that white light emission was obtained after $200 \mu \mathrm{L}$ of $\mathrm{Zn}^{2+}(0.1 \mathrm{M})$ was added. The CIE co-ordinate for the system also indicated the light could be tuned from natural white to cold white light. For instance, the CIE co-ordinate for the product obtained by the combination of $200 \mu \mathrm{L}$ of $\mathrm{Zn}^{2+}$ at $X$ $=0.354$ and $Y=0.3121$ did not demonstrate a significant difference from that of natural white light $(X=0.33, Y=0.33)$. Meanwhile, the CIE co-ordinate obtained from the addition of $250 \mu \mathrm{L}$ of $\mathrm{Zn}^{2+}$ at $X=0.3134$ and $Y=0.2803$ was typical cold white light. Since the product and fluorescence spectra of G-RCuNCs could be modified by tuning the amounts of G-CuNCs and other reagents, it can be concluded that other types of white light can also be obtained. Different from most white light-harvesting methods, this system does not require the introduction of other types of materials. ${ }^{32}$ Thus, this system provides a facile strategy for the generation of white light.

Besides, G-CuNCs and another product fabricated by the addition of a relatively high concentration of $\mathrm{Cu}^{2+}$ (G-R-CuNCs5) were investigated, as shown in Fig. S20.† It can be seen from Fig. S20a $†$ that the fluorescence emission intensity was significantly enhanced for G-CuNCs. This was similar to that in Fig. 7a. However, different from G-R-CuNCs-4, the red emission of G-R-CuNCs-5 was enhanced rather than quenched by the titration of $\mathrm{Zn}^{2+}$ (Fig. S20b $\dagger$ ). The comparison of G-R-CuNCs-4 and G-R-CuNCs-5 indicated that the red emission of the asprepared CuNCs could be either enhanced or quenched based on the synthetic conditions. When the product was fabricated with low amounts of $\mathrm{Cu}^{2+}$, the concentration of R-CuNCs was low. The red emission was mainly contributed by the FRET phenomenon. Also, the addition of $\mathrm{Zn}^{2+}$ inhibited R-CuNCs a

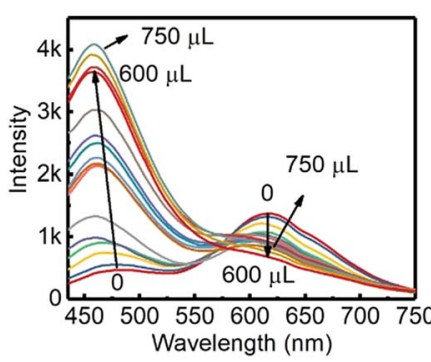

$\mathrm{b}$

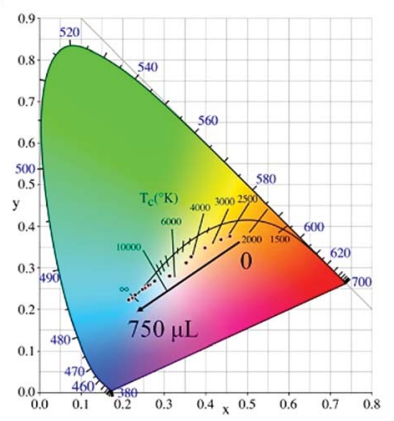

Fig. 7 (a) Fluorescence emission spectra of the as-prepared G-RCuNCs- 4 by the addition of different amounts of $\mathrm{Zn}^{2+}$ (with the slit widths of $10 \mathrm{~nm}$ for excitation and $5 \mathrm{~nm}$ for emission). Synthetic conditions: $500 \mu \mathrm{L}$ of G-CuNCs, $50 \mu \mathrm{L}$ of $0.079 \%$ THPS and $100 \mu \mathrm{L}$ of $0.01 \mathrm{M} \mathrm{Cu}^{2+}$. (b) Image of the CIE1931 chromaticity coordinate calculation for the corresponding samples. from getting close to G-RuNCs, which would decrease the energy transfer. Thus, a reversible phenomenon of FRET was observed (Fig. 7a). On the other hand, after the combination with a relatively high concentration of $\mathrm{Cu}^{2+}$, additional $\mathrm{R}$ CuNCs would be formed. The red emission was contributed both by FRET and the residual R-CuNCs that did not participate in the fluorescence enhancement process. It was demonstrated in previous report that $\mathrm{Zn}^{2+}$ could light up the red emission of GSH-CuNCs based on AIE. ${ }^{54}$ Meanwhile, the red emission for our product was enhanced due to AIE since the complex of $\mathrm{Zn}^{2+}$ with the carboxylate groups of the ligands rigidifies the $\mathrm{Cu}(\mathrm{I})$ thiolates, which restricted the intramolecular rotationvibrational motion. In summary, it can be concluded the fluorescence of the products can be controlled appropriately such as quenched, enhanced, or ratiometrically changed according to the corresponding applications and these processes can be well applied in different fields.

\section{Experimental}

\subsection{Chemical and materials}

Deionized water was used as the aqueous solution for dissolving all reagents. Copper(II) chloride $\left(\mathrm{CuCl}_{2}, 99 \%\right)$, L-ascorbic acid (LAA, $\geq 99 \%$ ), $( \pm)$ - $\alpha$-lipoic acid ( $\geq 99 \%$ ), polyvinylpyrrolidone (PVP, mol wt. 40 000), sodium hydroxide ( $\mathrm{NaOH}, \geq 98 \%$ ), bis [tetrakis(hydroxymethyl)phosphonium], sulfate solution (THPS, $70-75 \%$ in water) and sodium sulfide nonahydrate $\left(\mathrm{Na}_{2} \mathrm{~S}\right.$, $\geq 98 \%$ ) were purchased from Sigma Aldrich. Other reagents were all of analytical grade and obtained from Aladdin Company. All glassware was cleaned with aqua regia and rinsed with water prior to use.

\subsection{Synthesis of PVP-CuNCs}

PVP-protected CuNCs (PVP-CuNCs) were synthesized according to a previously reported paper with modifications. ${ }^{55}$ PVP $(0.5 \mathrm{~g})$ was added to a $20 \mathrm{~mL}$ water solution. After the complete dissolution, $2 \mathrm{~mL}$ of $0.1 \mathrm{M} \mathrm{CuCl} 2$ was injected. After the combination of $0.1 \mathrm{M}$ LAA, the mixer was shaken evenly. The reactants were transferred to an $80^{\circ} \mathrm{C}$ water bath and left for 3-5 hours until a light yellow color supernatant was observed.

\subsection{Synthesis of G-CuNCs}

$37.8 \mathrm{mg}$ of $( \pm)$ - $\alpha$-lipoic acid was added to a vial with $7.5 \mathrm{~mL}$ of water. $250 \mu \mathrm{L}$ of $1 \mathrm{M} \mathrm{NaOH}$ was added to the solution to dissolve $( \pm)$ - $\alpha$-lipoic acid to form DHLA. Further, $1 \mathrm{~mL}$ of the as-obtained PVP-CuNCs was added. Herein, the excess DHLA ligand in the colloid was further used as the protecting ligand for R-CuNCs. Thus, the as-formed colloid without separation was stored at $4{ }^{\circ} \mathrm{C}$ in a refrigerator for further use. For material characterization, G-CuNCs were purified by ultrafiltration with a $20 \mathrm{kDa}$ Millipore membrane. Besides this, G-CuNCs-2 were synthesized following the same procedure as that for G-CuNCs in the absence of PVP.

\subsection{Formation of G-R-CuNCs}

In a typical synthetic process, a certain amount of G-CuNCs were used. $100 \mu \mathrm{L}$ of $1 \mathrm{M} \mathrm{NaOH}$ was added to the solution. 
The total volume of the mixture was fixed as $2.5 \mathrm{~mL}$. Subsequently, $500 \mu \mathrm{L}$ of $0.079 \%$ THPS was added to the mixture. Finally, different amounts of $0.01 \mathrm{M} \mathrm{CuCl}_{2}$ solution was added to the above mixture. For the sample requiring a large amount of $\mathrm{Cu}^{2+}, 0.1 \mathrm{M}$ of $\mathrm{CuCl}_{2}$ was employed. In order to obtain the brightest red emission, F97 was employed to monitor the fluorescence emission spectra upon the addition of $\mathrm{Cu}^{2+}$ at 2 minutes intervals until the highest fluorescence emission intensity was reached. Then, a mixture of fluorescenceenhanced CuNCs (G-R-CuNCs) was obtained. After vacuum freeze drying, the as-obtained powder was preserved for longterm use. Besides, CuNCs with red emission was obtained with the assistance of G-CuNCs-2 (R-CuNCs-2) and the corresponding fluorescence-enhanced product (G-R-CuNCs-2) was fabricated.

\subsection{Synthesis of R-CuNCs-0}

$37.8 \mathrm{mg}$ of $( \pm)$ - $\alpha$-lipoic acid was added to a vial with $7.5 \mathrm{~mL}$ of water. $250 \mu \mathrm{L}$ of $1 \mathrm{M} \mathrm{NaOH}$ was dropped in the solution to dissolve ( \pm - $-\alpha$-lipoic acid to form DHLA. Next, $200 \mu \mathrm{L}$ of $0.1 \mathrm{M}$ $\mathrm{CuCl}_{2}$ and $500 \mu \mathrm{L}$ of $0.079 \%$ THPS was added to the above mixture. The mixture was stirred overnight until a light-yellow colloid with the strongest emission intensity was obtained. The sample were purified by ultrafiltration with a $20 \mathrm{kDa}$ Millipore membrane for characterization.

\subsection{Characterization}

All ultraviolet-visible (UV-Vis) absorption spectra were recorded using a UV-7504 UV-Vis spectrophotometer (Shanghai Xinmao). Fluorescence spectra were obtained on a fluorescence spectrophotometer (F97, Shanghai Lingguang). X-ray photoelectron spectroscopy (XPS) was performed on an ESCALAB 250 XI electron spectrometer (Thermo). The fluorescence lifetime was determined on a FL920 time-correlated single-photon-counting fluorescence lifetime spectrometer (Edinburgh Analytical Instruments, Edinburgh, U.K.). Transmission electron microscopy (TEM) images were obtained on a FEI Tecnai G2 F20 microscope. Dynamic light scattering (DLS) and zeta potential measurements were performed with a Malvern Zetasizer. FT-IR spectra were measured using an FT-IR spectrometer equipped with a DGTS detector (Nicolet is5/is10).

\subsection{Ratiometric sensing of $\mathbf{S}^{2-}$}

For $\mathrm{S}^{2-}$ analysis, $100 \mu \mathrm{L} 1 \mathrm{M} \mathrm{NaOH}$ was added to $2 \mathrm{~mL}$ of PBS buffer ( $\mathrm{pH}$ 8). After that, $150 \mu \mathrm{L}$ colloid of G-CuNCs was injected. Then, $20 \mu \mathrm{L}$ of $0.079 \%$ THPS was injected to the mixture. Next, $40 \mu \mathrm{L}$ of $0.01 \mathrm{M} \mathrm{Cu}^{2+}$ was added to the above solution and the $\mathrm{pH}$ value was tuned to $8 \mathrm{using} \mathrm{HCl}$. After mixing with $100 \mu \mathrm{L}$ of EDTA (50 mM), the sensor (G-CuNCs-3) in the absence of $\mathrm{S}^{2-}$ was used as the blank. Next, different concentrations $\mathrm{S}^{2-}$ were combined with the system for analysis of the calibration curve. Besides, other solutions containing amnion acids, GSH and cations were studied in the place of $\mathrm{S}^{2-}$ by measuring their fluorescence emission spectra for the selectivity study, which were recorded at an excitation wavelength of $400 \mathrm{~nm}$. All measurements were performed at room temperature.

\section{$3.8 \mathrm{~S}^{2-}$ sensing in real sample}

For the analysis of serum samples, chicken blood samples were collected from a local market. All the blood samples were centrifuged at $12000 \mathrm{rpm}$ for $15 \mathrm{~min}$ at $4{ }^{\circ} \mathrm{C}$. Then, the supernatant was subjected to 10 -fold dilution with $\mathrm{PBS}\left(10 \mathrm{mmol} \mathrm{L}^{-1}\right.$, $\mathrm{pH} 8$ ), and different concentrations of $\mathrm{S}^{2-}$ were added to the serum supernatant to prepare the spiked samples. Besides, all the samples were mixed with $100 \mu \mathrm{L}$ of EDTA $(50 \mathrm{mM})$. After incubation at room temperature for $2 \mathrm{~min}$, the resulting solution was diluted to the same volumes and investigated similarly to the standard samples.

\subsection{Fabrication of white light-emitting colloid}

$100 \mu \mathrm{L}$ of $1 \mathrm{M} \mathrm{NaOH}$ was added to $2 \mathrm{~mL}$ of water. Subsequently, $500 \mu \mathrm{L}$ colloid of G-CuNCs was injected. Then, $50 \mu \mathrm{L}$ of $0.079 \%$ THPS was injected to the mixture. Next, $100 \mu \mathrm{L}$ of $0.01 \mathrm{M} \mathrm{Cu}^{2+}$ was combined with the above solution and the product was obtained (G-CuNCs-4). Finally, $0.1 \mathrm{M}$ of $\mathrm{ZnSO}_{4}$ solution with different volumes was added to the system when the total volume was fixed. F97 was used to measure the fluorescence emission spectra and CIE1931 was employed to analyze the CIE of the light.

\section{Conclusions}

The strategy of using green emitting copper nanoclusters as assistants for the fabrication of red emitting copper nanoclusters was demonstrated. This method for the fabrication of the product not only provides a facile protocol for the fast generation of nanoclusters with much brighter fluorescence with assistance from the nanoclusters themselves, but also can provide avenues for future ratio-metric analysis with all-copper nanoclusters. Additionally, it has potential for the development of light-harvesting materials via a simple process. The asobtained products of all-copper nanoclusters are excellent materials for application in different fields.

\section{Conflicts of interest}

There are no conflicts to declare.

\section{Acknowledgements}

We are grateful for the financial support from the National Natural Science Foundation of China (Grant No. 81702105 and No. 81671742) and Liaoning (Grant No. 201602288).

\section{Notes and references}

1 S. Xu, Y. Nie, L. Jiang, J. Wang, G. Xu, W. Wang and X. Luo, Anal. Chem., 2018, 90, 4039-4045.

2 L. Y. Chen, C. W. Wang, Z. Yuan and H. T. Chang, Anal. Chem., 2015, 87, 216-229.

3 N. Goswami, Q. Yao, Z. Luo, J. Li, T. Chen and J. Xie, J. Phys. Chem. Lett., 2016, 7, 962-975. 
4 R. Tian, S. Zhang, M. Li, Y. Zhou, B. Lu, D. Yan, M. Wei, D. G. Evans and X. Duan, Adv. Funct. Mater., 2015, 25, 5006-5015.

5 N. Goswami, F. Lin, Y. Liu, D. T. Leong and J. Xie, Chem. Mater., 2016, 28, 4009-4016.

6 Z. Wu, H. Liu, T. Li, J. Liu, J. Yin, O. F. Mohammed, O. M. Bakr, Y. Liu, B. Yang and H. Zhang, J. Am. Chem. Soc., 2017, 139, 4318-4321.

7 M. Zhao, Z. Qian, M. Zhong, Z. Chen, H. Ao and H. Feng, ACS Appl. Mater. Interfaces, 2017, 9, 32887-32895.

8 M. T. Hembury, N. Beztsinna, H. Asadi, J. van den Dikkenberg, J. D. Meeldijk, W. E. Hennink and T. Vermonden, Biomacromolecules, 2018, 19(7), 2841-2848.

9 Z. Wu, J. Liu, Y. Gao, H. Liu, T. Li, H. Zou, Z. Wang, K. Zhang, Y. Wang, H. Zhang and B. Yang, J. Am. Chem. Soc., 2015, 137, 12906-12913.

10 K. Pyo, V. D. Thanthirige, K. Kwak, P. Pandurangan, G. Ramakrishna and D. Lee, J. Am. Chem. Soc., 2015, 137, 8244-8250.

11 F. Cao, E. Ju, C. Liu, W. Li, Y. Zhang, K. Dong, Z. Liu, J. Ren and X. Qu, Nanoscale, 2017, 9, 4128-4134.

12 X. Su and J. Liu, ACS Appl. Mater. Interfaces, 2017, 9, 39023910.

13 T. Y. Zhou, L. P. Lin, M. C. Rong, Y. Q. Jiang and X. Chen, Anal. Chem., 2013, 85, 9839-9844.

14 P. C. Chen, Y. C. Li, J. Y. Ma, J. Y. Huang, C. F. Chen and H. T. Chang, Sci. Rep., 2016, 6, 24882.

15 X. Jia, J. Li and E. Wang, Small, 2013, 9, 3873-3879.

16 S. Y. Tao Chen, J. Chai, Y. Song, J. Fan, B. Rao, H. Sheng and H. Yu, Sci. Adv., 2017, 3, e1700956.

17 Y. Huang, H. Feng, W. Liu, S. Zhang, C. Tang, J. Chen and Z. Qian, J. Mater. Chem. B, 2017, 5, 5120-5127.

18 D. Li, Z. Chen and X. Mei, Adv. Colloid Interface Sci., 2017, 250, 25-39.

19 Y. Lu, W. Wei and W. Chen, Chin. Sci. Bull., 2012, 57, 41-47.

20 J. Zhao and R. Jin, Nano Today, 2018, 18, 86-102.

21 X. Yang, J.-K. Sun, M. Kitta, H. Pang and Q. Xu, Nat. Catal., 2018, 1, 214-220.

22 L. Liu and A. Corma, Chem. Rev., 2018, 118(10), 4981-5079.

23 Z. Wu, Y. Li, J. Liu, Z. Lu, H. Zhang and B. Yang, Angew. Chem., Int. Ed., 2014, 53, 12196-12200.

24 H. K. Masaki Takahashi, W.-J. Chun, M. Kori, T. Imaoka and K. Yamamoto, Sci. Adv., 2017, 3, e1700101.

25 C. Wang, C. Wang, L. Xu, H. Cheng, Q. Lin and C. Zhang, Nanoscale, 2014, 6, 1775-1781.

26 N. Vilar-Vidal, J. Rivas and M. A. López-Quintela, ACS Catal., 2012, 2, 1693-1697.

27 L.-L. Wang, J. Qiao, H.-H. Liu, J. Hao, L. Qi, X.-P. Zhou, D. Li, Z.-X. Nie and L.-Q. Mao, Anal. Chem., 2014, 86, 9758-9764.

28 J. T. Del Bonis-O'Donnell, A. Thakrar, J. W. Hirschberg, D. Vong, B. N. Queenan, D. K. Fygenson and S. Pennathur, ACS Chem. Neurosci., 2018, 9, 849-857.

29 Q. Yu, P. Gao, K. Y. Zhang, X. Tong, H. Yang, S. Liu, J. Du, Q. Zhao and W. Huang, Light: Sci. Appl., 2017, 6, e17107.

30 Y. Yan, H. Yu, K. Zhang, M. Sun, Y. Zhang, X. Wang and S. Wang, Nano Res., 2016, 9, 2088-2096.
31 J. G. C. Shahad, K. Alsaiari, M. Hammami, H. W. Omar and P. Neelakanda, Adv. Healthcare Mater., 2017, 6, 1601135.

32 M. K. Barman, B. Paramanik, D. Bain and A. Patra, Chemistry, 2016, 22, 11699-11705.

33 D. Li, Z. Chen, Z. Wan, T. Yang, H. Wang and X. Mei, RSC Adv., 2016, 6, 34090-34095.

34 A. D. Upashi Goswam, A. Raza, R. Kandimalla, S. Kalita, S. Ghosh and A. Chattopadhyay, ACS Appl. Mater. Interfaces, 2018, 10, 3282-3294.

35 Y. Raju, P. Krishnamurthi, P. L. Paulose and P. T. Manoharan, Nanoscale, 2017, 9, 17963-17974.

36 L. Shang, L. Yang, F. Stockmar, R. Popescu, V. Trouillet, M. Bruns, D. Gerthsen and G. U. Nienhaus, Nanoscale, 2012, 4, 4155-4160.

37 J. P. Otto, L. Wang, I. Pochorovski, S. M. Blau, A. AspuruGuzik, Z. Bao, G. S. Engel and M. Chiu, Chem. Sci., 2018, 9, 3694-3703.

38 Z. Luo, X. Yuan, Y. Yu, Q. Zhang, D. T. Leong, J. Y. Lee and J. Xie, J. Am. Chem. Soc., 2012, 134, 16662-16670.

39 D. Li, Z. Chen, T. Yang, H. Wang, N. Lu and X. Mei, RSC Adv., 2016, 6, 19182-19189.

40 Y. Liu, D. Ding, Y. Zhen and R. Guo, Biosens. Bioelectron., 2017, 92, 140-146.

41 Z. Wang, A. S. Susha, B. Chen, C. Reckmeier, O. Tomanec, R. Zboril, H. Zhong and A. L. Rogach, Nanoscale, 2016, 8, 7197-7202.

42 J. Jiang, C. V. Conroy, M. M. Kvetny, G. J. Lake, J. W. Padelford, T. Ahuja and G. Wang, J. Phys. Chem. C, 2014, 118, 20680-20687.

43 M. Porta, M. T. Nguyen, Y. Ishida and T. Yonezawa, RSC Adv., 2016, 6, 105030-105034.

44 C. Huang, Q. Liu, W. Fan and X. Qiu, Sci. Rep., 2015, 5, 16736.

45 Y. Wang, A. V. Biradar, G. Wang, K. K. Sharma, C. T. Duncan, S. Rangan and T. Asefa, Chemistry, 2010, 16, 10735-10743.

46 N. K. Das, S. Ghosh, A. Priya, S. Datta and S. Mukherjee, J. Phys. Chem. C, 2015, 119, 24657-24664.

47 A. Baral, K. Basu, S. Ghosh, K. Bhattacharyya, S. Roy, A. Datta and A. Banerjee, Nanoscale, 2017, 9, 4419-4429.

48 S. A. Crooker, J. A. Hollingsworth, S. Tretiak and V. I. Klimov, Phys. Rev. Lett., 2002, 89, 186802.

49 J. F. M. Ranojoy Bose, J. Gao, K. M. Rickey, C. J. Chen, D. V. Talapin, C. B. Murray and A. C. W. Won, Nano Lett., 2008, 8, 2006-2011.

50 S. Maity, D. Bain, K. Bhattacharyya, S. Das, R. Bera, B. Jana, B. Paramanik, A. Datta and A. Patra, J. Phys. Chem. C, 2017, 122, 13354-13362.

51 X. C. Jeremy Essner, T. Wood and G. Baker, Analyst, 2018, 143, 1036-1041.

52 K. Basu, K. Gayen, T. Mitra, A. Baral, S. S. Roy and A. Banerjee, ChemNanoMat, 2017, 3, 808-814.

53 X. Kang, H. Chong and M. Zhu, Nanoscale, 2018, 10, 1075810834.

54 L. Lin, Y. Hu, L. Zhang, Y. Huang and S. Zhao, Biosens. Bioelectron., 2017, 94, 523-529.

55 Y. E. Shi, S. Luo, X. Ji, F. Liu, X. Chen, Y. Huang, L. Dong and L. Wang, Dalton Trans., 2017, 46, 14251-14255. 